\title{
A Prospective Study of Analysis of Correlation of Abo Blood Groups with Coronary Artery Disease
}

\author{
Dr.V.Ushapadmini M.D, Dr.Manohari Ramachandran M.D, \\ ${ }^{I}$ Department Of Medicine,Coimbatore Medical College,Coimbatore,The Tamilnadu Dr..M.G.R.Medical \\ University, Indiaauthor \\ ${ }^{2}$ Department Of Medicine,Ciimbatore Medical College,The Tamilnadu Dr.M.G.R.Medical University,India
}

\begin{abstract}
A B O$ Blood group system is a well known risk factor clinically linked to thrombotic vascular diseases.Since 1901 When DR.Carl Landsteiner discovered the Blood groups it has been linked with Cognitive Impairment,Preeclampsia,Bleeding and Longevity.ABO Blood group may also be related to caisation of Coronary artery disease. We did a Prospective study on Patients Who were admitted to the Intensive Coronary Care Unit Of Coimbatore medical College Hospital And tried to correlate the Various Blood Groups with Coronary Artery Disease

Keywords: Blood Groups, Coronary Artery Disease, Complications, Risk Factors in MI,Thrombotic Factor-
\end{abstract}

\section{Introduction}

The current study was aimed to analyse the common ABO blood groups associated with Coronary Artery disease

Aim Of The Study: To Examine Patients admitted in the ICCU with chest pain

To analyse whether they are Hypertensive,Diabetic,Smokers,Alcoholics

To Check their ABO Blood groups

To analyse the Complications encountered during their stay

Materials And Methods: The present study was conducted at Coimbatore Medical College Between January 2016 to June 2016 for a period of 6 months as an observational study

Inclusion Criteria: All patients with chest pain and diagnosed to have Coronary artery disease while admitted to ICCU were included

Exclusion Criteria: Those patients who were admitted but found to have Non cardiac chest pain were excluded from the study A Detailed History regarding chest pain and risk factors like Hypertension,Diabetes Mellitus,Smokind,Alcoholism was taken

Investigations done 12 lead ECG and $\mathrm{ABO}$ blood grouping done

\section{Results And Observation}

Age group analysis-50\% of patients belonged to $40-60$ yrs age group,29\% of patients belonged to 60 80 yrs age group Sex distribution- $80 \%$ males and 19\% females Hypertension-33.3\% were hypertensive and 66.7\% were non hypertensive Diabetes-27.7\% were diabetic and $72.3 \%$ were non diabetic Smokers- $62.4 \%$ were smokers and $37.6 \%$ were not smokers Alcoholics-41.8\% were alcoholics and 58.2\% were not alcoholics Prevalence of blood groups among Myocardial Infarction patients-

$\mathrm{B}+$ was the commonest blood group followed by $\mathrm{O}+$ and $\mathrm{A}+$ to cause $\mathrm{MI}$

Proportion of patients who developed complications-

7.1\% had developed complications of which Complete heart block followed by LBBB and RBBB

4 patients from blood groups $\mathrm{O}+$ and $\mathrm{B}+$ developed complications

\section{Conclusions}

1) The commonest age group was $40-60$ yrs to develop Myocardial Infarction

2) Male sex was predominantly affected

3) $30 \%$ patients had risk factors of Hypertension/Diabetes mellitus 
4) $60 \%$ patients were smokers

5) The commonest $\mathrm{ABO}$ Blood group implicated was $\mathrm{B}+$ followed by $\mathrm{O}+$ and $\mathrm{A}+$ blood groups

6) The commonest complication was Complete Heart Block followed by Bundle branch blocks

7) Anterior wall MI was the commonest followed by Inferior wall MI

\section{Bibliography}

1) $\mathrm{ABO}$ Blood group system and Coronary artery disease-An updated systematic review and Meta analysisZhuo Chen

2)ABO Blood groups and Coronary artery disease-A systematic review and Meta analysis-Wu,Bayoumi ,Vickers and clark

3)Association of Blood group A in coronary artery disease in Young adults in Taiwan-Lee,Lin,Wang and Chang

4) $\mathrm{ABO}$ Blood groups and coronary artery disease-atvb.ahajournals.org

5) $\mathrm{ABO}$ Blood group alleles and risk of CAD-www.sciencedirect.com

6) $\mathrm{ABO}$ blood group and coronary artery disease in Iranian patients-labmed.oxfordjournals.org

7)Blood group and risk of CAD-www.livescience.com

Table 1: Background characteristics:

\begin{tabular}{|c|c|c|c|}
\hline S.No & Particulars & Number $(N=141)$ & Percentage (\%) \\
\hline \multirow[t]{5}{*}{1} & Age Group In Years & & \\
\hline & $20-40$ & 27 & 19.1 \\
\hline & $40-60$ & 71 & 50.4 \\
\hline & $60-80$ & 42 & 29.8 \\
\hline & $>80$ & 1 & 0.7 \\
\hline \multirow[t]{3}{*}{2} & Sex & & \\
\hline & Male & 114 & 80.9 \\
\hline & Female & 27 & 19.1 \\
\hline \multirow[t]{3}{*}{3} & Hypertension & & \\
\hline & Yes & 47 & 33.3 \\
\hline & No & 94 & 66.7 \\
\hline \multirow[t]{3}{*}{4} & Diabetes & & \\
\hline & Yes & 39 & 27.7 \\
\hline & No & 102 & 72.3 \\
\hline \multirow[t]{3}{*}{5} & Smoking & & \\
\hline & Yes & 88 & 62.4 \\
\hline & No & 53 & 37.6 \\
\hline \multirow[t]{3}{*}{6} & Alcohol Use & & \\
\hline & Yes & 59 & 41.8 \\
\hline & No & 82 & 58.2 \\
\hline
\end{tabular}

Table 2:

\begin{tabular}{|l|l|l|l|}
\hline S.No & Blood Group & Number $(\mathbf{N}=\mathbf{1 4 1})$ & Percentage $\mathbf{( \% )}$ \\
\hline 1 & O+ & 39 & 27.7 \\
\hline 2 & A+ & 29 & 20.6 \\
\hline 3 & B + & 55 & 39.0 \\
\hline 4 & Ab+ & 8 & 5.7 \\
\hline 5 & O- & 3 & 2.1 \\
\hline 6 & A- & 4 & 2.8 \\
\hline 7 & B- & 2 & 1.4 \\
\hline 8 & Ab- & 1 & 0.7 \\
\hline & & & \\
\hline
\end{tabular}

Patients with B+ $(39 \%)$ Blood group are more prone to develop MI, followed by those with $\mathrm{O}+(27.7)$ and $\mathrm{A}+$ (20.6)

Table 3: Proportion of MI patients who developed any one complication

\begin{tabular}{|l|l|l|l|}
\hline$\bullet \quad$ S.No & Comlication & Number $(\mathbf{N}=\mathbf{1 4 1})$ & Percentage $(\%)$ \\
\hline 1 & Yes & 10 & 7.1 \\
\hline 2 & No & 131 & 92.9 \\
\hline
\end{tabular}


Table 4: Complication:

\begin{tabular}{|l|l|l|l|}
\hline S.No & Type Of Complication & Number $(\mathbf{N}=10) *$ & Percentage \\
\hline 1 & Heart Block & 1 & 10 \\
\hline 2 & Complete Block & 2 & 20 \\
\hline 3 & Vsr & 1 & 10 \\
\hline 4 & Lbbb & 2 & 20 \\
\hline 5 & Rbbb & 2 & 20 \\
\hline 6 & Hocm & 1 & 10 \\
\hline 7 & Old Iwmi & 1 & 10 \\
\hline
\end{tabular}

*131 had no complications. Only 10 patients had complication

Table 5: Complications and blood group of patients

\begin{tabular}{|c|c|c|c|c|}
\hline & & \multicolumn{2}{|c|}{ Complication } & \multirow[t]{2}{*}{ Total } \\
\hline & & yes & No & \\
\hline \multirow[t]{8}{*}{ bloodgp } & $\mathrm{O}+$ & 4 & 35 & 39 \\
\hline & $\mathrm{A}+$ & 2 & 27 & 29 \\
\hline & $\mathrm{B}+$ & 4 & 51 & 55 \\
\hline & $\mathrm{AB}+$ & 0 & 8 & 8 \\
\hline & A- & 0 & 4 & 4 \\
\hline & B- & 0 & 2 & 2 \\
\hline & $0-$ & 0 & 3 & 3 \\
\hline & AB- & 0 & 1 & 1 \\
\hline \multicolumn{2}{|l|}{ Total } & 10 & 131 & 141 \\
\hline
\end{tabular}

4 patients each from $0+$ and $\mathrm{B}+$ developed complications. 2 patients having $\mathrm{A}+$ blood group had complications. 1 patient from $\mathrm{AB}$ - had complications.

Table 5: Complication:

\begin{tabular}{|l|l|l|l|}
\hline S.No & Type Of Complication & Number $(\mathbf{N}=10$ & $*$ \\
\hline 1 & Heart Block & 1 & 10 \\
\hline 2 & Complete Block & 2 & 20 \\
\hline 3 & Vsr & 1 & 10 \\
\hline 4 & Lbbb & 2 & 20 \\
\hline 5 & Rbbb & 2 & 20 \\
\hline 6 & Hocm & 1 & 10 \\
\hline 7 & Old Iwmi & 1 & 10 \\
\hline
\end{tabular}

*131 had no complications. Only 10 patients had complication

\section{Statistics}

\begin{tabular}{|l|l|l|l|l|l|l|l|l|l|}
\hline \multicolumn{2}{|c|}{} & age group & sex & $\begin{array}{l}\text { hypertensi } \\
\text { on }\end{array}$ & $\begin{array}{l}\text { diabete } \\
\text { s }\end{array}$ & smoking & alcohol & $\begin{array}{l}\text { Complicatio } \\
\text { n }\end{array}$ & bloodgp \\
\hline N & Valid & 138 & 141 & 140 & 141 & 141 & 141 & 138 & 140 \\
\hline & $\begin{array}{l}\text { Missin } \\
\text { g }\end{array}$ & 4 & 1 & 2 & 1 & 1 & 1 & 4 & 2 \\
\hline
\end{tabular}

\section{AGe group}

\begin{tabular}{|c|c|c|c|c|c|}
\hline & & Frequency & Percent & Valid Percent & $\begin{array}{l}\text { Cumulative } \\
\text { Percent }\end{array}$ \\
\hline \multirow[t]{7}{*}{ Valid } & $30-40$ & 24 & 16.9 & 17.4 & 17.4 \\
\hline & $40-50$ & 38 & 26.8 & 27.5 & 44.9 \\
\hline & $50-60$ & 33 & 23.2 & 23.9 & 68.8 \\
\hline & $60-70$ & 30 & 21.1 & 21.7 & 90.6 \\
\hline & $70-80$ & 12 & 8.5 & 8.7 & 99.3 \\
\hline & $>80$ & 1 & .7 & .7 & 100.0 \\
\hline & Total & 138 & 97.2 & 100.0 & \\
\hline Missing & System & 4 & 2.8 & & \\
\hline \multicolumn{2}{|l|}{ Total } & 142 & 100.0 & & \\
\hline
\end{tabular}

sex

\begin{tabular}{|l|l|l|l|l|l|}
\hline \multicolumn{2}{|l|}{} & Frequency & Percent & Valid Percent & $\begin{array}{l}\text { Cumulative } \\
\text { Percent }\end{array}$ \\
\hline Valid & male & 114 & 80.3 & 80.9 & 80.9 \\
\hline & female & 27 & 19.0 & 19.1 & 100.0 \\
\hline & Total & 141 & 99.3 & 100.0 & \\
\hline Missing & System & 1 & .7 & & \\
\hline
\end{tabular}


A Prospective Study Of Analysis Of Correlation Of Abo Blood Groups With Coronary..

\begin{tabular}{|c|c|c|c|c|c|}
\hline \multicolumn{2}{|c|}{ Total } & 142 & & & \\
\hline \multicolumn{6}{|c|}{ hypertension } \\
\hline & & Frequency & Percent & Valid Percent & $\begin{array}{l}\text { Cumulative } \\
\text { Percent }\end{array}$ \\
\hline \multirow[t]{3}{*}{ Valid } & yes & 47 & 33.1 & 33.6 & 33.6 \\
\hline & no & 93 & 65.5 & 66.4 & 100.0 \\
\hline & Total & 140 & 98.6 & 100.0 & \\
\hline Missing & System & 2 & 1.4 & & \\
\hline \multicolumn{2}{|l|}{ Total } & 142 & \multicolumn{2}{|l|}{100.0} & \\
\hline
\end{tabular}

diabetes

\begin{tabular}{|ll|l|l|l|l|}
\hline & & & & & $\begin{array}{l}\text { Cumulative } \\
\text { Percent }\end{array}$ \\
\hline Valid & yes & 39 & Frequency & Percent & Valid Percent \\
& no & 102 & 27.5 & 27.7 & 27.7 \\
& Total & 141 & 91.8 & 72.3 & 100.0 \\
Missing & System & 1 & .7 & 100.0 & \\
Total & & 142 & 100.0 & & \\
\hline
\end{tabular}

\begin{tabular}{|ll|l|l|l|l|}
\hline \multicolumn{1}{|c|}{ smoking } & & & $\begin{array}{l}\text { Cumulative } \\
\text { Percent }\end{array}$ \\
\hline Valid & yes & 88 & Frequency & Percent & Valid Percent \\
& no & 53 & 62.0 & 62.4 & 62.4 \\
& Total & 141 & 37.3 & 37.6 & 100.0 \\
Missing & System & 1 & 99.3 & 100.0 & \\
Total & & 142 & .7 & & \\
\hline
\end{tabular}

\begin{tabular}{|ll|l|l|l|l|}
\multicolumn{1}{|c}{ alcohol } \\
& & & & Cumulative \\
\multicolumn{1}{|l|}{} & Frequency & Percent & Valid Percent & Percent \\
\hline Valid & yes & 59 & 41.5 & 41.8 & 41.8 \\
& no & 82 & 57.7 & 58.2 & 100.0 \\
& Total & 141 & 99.3 & 100.0 & \\
Missing & System & 1 & .7 & & \\
Total & & 142 & 100.0 & & \\
\hline
\end{tabular}

\section{Complication}

\begin{tabular}{|l|l|l|l|l|l|}
\hline \multicolumn{2}{|c|}{} & Frequency & Percent & Valid Percent & $\begin{array}{l}\text { Cumulative } \\
\text { Percent }\end{array}$ \\
\hline \multirow{3}{*}{ Valid } & yes & 10 & 7.0 & 7.2 & 7.2 \\
\cline { 2 - 6 } & no & 128 & 90.1 & 92.8 & 100.0 \\
\cline { 2 - 6 } & Total & 138 & 97.2 & 100.0 & \\
\hline Missing & System & 4 & 2.8 & & \\
\hline \multicolumn{2}{|l|}{ Total } & 142 & 100.0 & & \\
\hline
\end{tabular}

bloodgp

\begin{tabular}{|l|l|l|l|l|l|}
\hline \multicolumn{2}{|c|}{} & Frequency & Percent & Valid Percent & $\begin{array}{l}\text { Cumulative } \\
\text { Percent }\end{array}$ \\
\hline \multirow{4}{*}{ Valid } & O+ & 38 & & & 27.1 \\
\cline { 2 - 6 } & A+ & 29 & 26.8 & 27.1 & 47.9 \\
\cline { 2 - 6 } & B + & 54 & 20.4 & 20.7 & 86.4 \\
\cline { 2 - 6 } & AB+ & 8 & 38.0 & 38.6 & 92.1 \\
\cline { 2 - 6 } & A- & 4 & 5.6 & 5.7 & 95.0 \\
\cline { 2 - 6 } & B- & 2 & 2.8 & 2.9 & 96.4 \\
\cline { 2 - 6 } & $0-$ & 3 & 1.4 & 1.4 & 98.6 \\
\cline { 2 - 6 } & AB- & 1 & 2.1 & 2.1 & 99.3 \\
\cline { 2 - 6 } & 14.00 & 1 & .7 & .7 & 100.0 \\
\cline { 2 - 6 } & Total & 140 & 98.6 & 100.0 & \\
\hline
\end{tabular}


A Prospective Study Of Analysis Of Correlation Of Abo Blood Groups With Coronary..

\begin{tabular}{|c|c|c|c|c|c|}
\hline Missing & System & 2 & 1.4 & & \\
\hline \multicolumn{2}{|l|}{ Total } & 142 & 100.0 & & \\
\hline \multicolumn{6}{|c|}{ diagnosis } \\
\hline & & Frequency & Percent & Valid Percent & $\begin{array}{l}\text { Cumulative } \\
\text { Percent }\end{array}$ \\
\hline \multirow[t]{14}{*}{ Valid } & AWMI & 75 & 52.8 & 53.2 & 53.2 \\
\hline & IW/RV/PW & 3 & 2.1 & 2.1 & 55.3 \\
\hline & IVMI & 42 & 29.6 & 29.8 & 85.1 \\
\hline & IW/RW & 3 & 2.1 & 2.1 & 87.2 \\
\hline & IW/RV & 2 & 1.4 & 1.4 & 88.7 \\
\hline & NSTEMI & 1 & .7 & .7 & 89.4 \\
\hline & IWMI/RV & 1 & .7 & .7 & 90.1 \\
\hline & HLWMI & 1 & .7 & .7 & 90.8 \\
\hline & IW/PW/RV & 2 & 1.4 & 1.4 & 92.2 \\
\hline & IWMI/PV/RW & 1 & .7 & .7 & 92.9 \\
\hline & ASMI & 4 & 2.8 & 2.8 & 95.7 \\
\hline & IW/RV/PV & 3 & 2.1 & 2.1 & 97.9 \\
\hline & ALMI & 3 & 2.1 & 2.1 & 100.0 \\
\hline & Total & 141 & 99.3 & 100.0 & \\
\hline Missing & System & 1 & .7 & & \\
\hline \multicolumn{2}{|l|}{ Total } & 142 & 100.0 & & \\
\hline
\end{tabular}

Correlations

\begin{tabular}{|ll|l|l|}
\hline & & diagnosis & bloodgp \\
\hline diagnosis & Pearson Correlation & 1 & .022 \\
& Sig. (2-tailed) & & .797 \\
& N & 141 & 140 \\
bloodgp & Pearson Correlation & .022 & 1 \\
& Sig. (2-tailed) & .797 & \\
& N & 140 & 140 \\
\hline
\end{tabular}

bloodgp * sex Crosstabulation

Count

\begin{tabular}{|ll|l|l|l|}
\hline & & \multicolumn{2}{l|}{ sex } & Total \\
\cline { 3 - 5 } & male & female & male \\
\hline bloodgp & O+ & 29 & 9 & 38 \\
& A+ & 26 & 3 & 29 \\
& B+ & 44 & 10 & 54 \\
& AB+ & 8 & 0 & 8 \\
& A- & 2 & 2 & 4 \\
& B- & 2 & 0 & 2 \\
& $0-$ & 0 & 3 & 3 \\
& AB- & 1 & 0 & 1 \\
Total & 14.00 & 1 & 0 & 1 \\
\hline
\end{tabular}

Chi-Square Tests

\begin{tabular}{|l|l|l|l|}
\hline & Value & $\mathrm{df}$ & $\begin{array}{l}\text { Asymp. Sig. } \\
\text { (2-sided) }\end{array}$ \\
\hline Pearson Chi-Square & $19.829(\mathrm{a})$ & 8 & .011 \\
Likelihood Ratio & 19.106 & 8 & .014 \\
Linear-by-Linear & .507 & 1 & .476 \\
Association & 140 & & \\
N of Valid Cases & & &
\end{tabular}

a 11 cells $(61.1 \%)$ have expected count less than 5 . The minimum expected count is .19. 


\section{Symmetric Measures}

\begin{tabular}{|l|l|l|l|l|l|}
\hline \multicolumn{2}{|l|}{} & Value & $\begin{array}{l}\text { Asymp. Std. } \\
\text { Error(a) }\end{array}$ & Approx. T(b) & Approx. Sig. \\
\hline Interval by Interval & Pearson's R & .060 & .094 & .711 & $.478(\mathrm{c})$ \\
\hline Ordinal by Ordinal & $\begin{array}{l}\text { Spearman } \\
\text { Correlation }\end{array}$ & .023 & .093 & .275 & $.783(\mathrm{c})$ \\
\hline N of Valid Cases & 140 & & & \\
\hline
\end{tabular}

a Not assuming the null hypothesis.

b Using the asymptotic standard error assuming the null hypothesis.

c Based on normal approximation.

bloodgp * diagnosis Crosstabulation

Count

\begin{tabular}{|l|l|l|l|l|l|l|l|l|l|l|l|l|l|l|l|}
\hline \multicolumn{2}{|l|}{ Diagnosis } & \begin{tabular}{l} 
AWMI \\
\multicolumn{2}{|l|}{}
\end{tabular} & $\begin{array}{l}\text { IW/R } \\
\text { V/P }\end{array}$ & $\begin{array}{l}\text { IVM } \\
\text { I }\end{array}$ & $\begin{array}{l}\text { IW/R } \\
\text { W }\end{array}$ & $\begin{array}{l}\text { IW/ } \\
\text { RV }\end{array}$ & $\begin{array}{l}\text { NSTE } \\
\text { MI }\end{array}$ & $\begin{array}{l}\text { IWMI } \\
\text { RV }\end{array}$ & HLWMI & $\begin{array}{l}\text { IW/PW } \\
\text { RV }\end{array}$ & $\begin{array}{l}\text { IWMIP } \\
\text { V/RW }\end{array}$ & $\begin{array}{l}\text { AS } \\
\text { MI }\end{array}$ & $\begin{array}{l}\text { IW/RV } \\
\text { /PV }\end{array}$ & $\begin{array}{l}\text { AL } \\
\text { MI }\end{array}$ & $\begin{array}{l}\text { AWM } \\
\text { I }\end{array}$ \\
\hline $\begin{array}{l}\text { Blood } \\
\text { gp }\end{array}$ & $0+$ & 22 & 0 & 10 & 1 & 0 & 1 & 0 & 0 & 0 & 1 & 1 & 1 & 1 & 38 \\
\hline & $\mathrm{A}+$ & 15 & 0 & 11 & 0 & 0 & 0 & 0 & 1 & 0 & 0 & 0 & 1 & 1 & 29 \\
\hline & $\mathrm{B}+$ & 28 & 2 & 15 & 1 & 1 & 0 & 1 & 0 & 1 & 0 & 3 & 1 & 1 & 54 \\
\hline & $\mathrm{AB}+$ & 6 & 0 & 2 & 0 & 0 & 0 & 0 & 0 & 0 & 0 & 0 & 0 & 0 & 8 \\
\hline & $\mathrm{A}-$ & 1 & 1 & 2 & 0 & 0 & 0 & 0 & 0 & 0 & 0 & 0 & 0 & 0 & 4 \\
\hline & $\mathrm{B}-$ & 1 & 0 & 0 & 0 & 1 & 0 & 0 & 0 & 0 & 0 & 0 & 0 & 0 & 2 \\
\hline & $0-$ & 0 & 0 & 2 & 0 & 0 & 0 & 0 & 0 & 1 & 0 & 0 & 0 & 0 & 3 \\
\hline & $\mathrm{AB}-$ & 1 & 0 & 0 & 0 & 0 & 0 & 0 & 0 & 0 & 0 & 0 & 0 & 0 & 1 \\
\hline & 14.00 & 0 & 0 & 0 & 1 & 0 & 0 & 0 & 0 & 0 & 0 & 0 & 0 & 0 & 1 \\
\hline Total & & 74 & 3 & 42 & 3 & 2 & 1 & 1 & 1 & 2 & 1 & 4 & 3 & 3 & 140 \\
\hline
\end{tabular}

Chi-Square Tests

\begin{tabular}{|l|l|l|l|}
\hline & Value & df & $\begin{array}{l}\text { Asymp. Sig. (2- } \\
\text { sided) }\end{array}$ \\
\hline Pearson Chi-Square & $137.835(\mathrm{a})$ & 96 & .003 \\
\hline Likelihood Ratio & 56.477 & 96 & 1.000 \\
\hline $\begin{array}{l}\text { Linear-by-Linear } \\
\text { Association }\end{array}$ & .067 & 1 & .796 \\
\hline N of Valid Cases & 140 & & \\
\hline
\end{tabular}

a 111 cells $(94.9 \%)$ have expected count less than 5 . The minimum expected count is .01.

\begin{tabular}{|l|l|l|l|l|l|}
\hline \multicolumn{2}{|l|}{} & Vymmetric Measures \\
\hline Interval by Interval & $\begin{array}{l}\text { Pearson's } \\
\mathrm{R}\end{array}$ & $\begin{array}{l}\text { Asymp. Std. } \\
\text { Error(a) }\end{array}$ & Approx. T(b) & Approx. Sig. \\
\hline Ordinal by Ordinal & $\begin{array}{l}\text { Spearman } \\
\text { Correlatio } \\
\mathrm{n}\end{array}$ & .047 & .068 & .258 & $.797(\mathrm{c})$ \\
\hline N of Valid Cases & 140 & .085 & .556 & $.579(\mathrm{c})$ \\
\hline
\end{tabular}

a Not assuming the null hypothesis.

b Using the asymptotic standard error assuming the null hypothesis.

c Based on normal approximation. 